\title{
Protocol for a Systematic Review Exploring the Psychometric Properties of Health and Wellbeing Measures Used With Adolescents Experiencing Intellectual Disabilities.
}

\section{Stephanie Maguire ( $\nabla$ s.maguire1@ulster.ac.uk)}

Ulster University - Coleraine Campus: Ulster University https://orcid.org/0000-0002-3834-4912

Jenny Davison

Ulster University - Coleraine Campus: Ulster University

Marian McLaughlin

Ulster University - Coleraine Campus: Ulster University

Victoria Simms

Ulster University - Coleraine Campus: Ulster University

\section{Protocol}

Keywords: Health, Wellbeing, Quality of life, Adolescence, Intellectual Disability, Measures, Psychometric, Validity, Reliability

Posted Date: April 9th, 2021

DOl: https://doi.org/10.21203/rs.3.rs-397339/v1

License: (c) (i) This work is licensed under a Creative Commons Attribution 4.0 International License.

Read Full License 


\section{Abstract \\ Background}

Whilst there are studies that have systematically reviewed the psychometric properties of Quality-of-Life measures for children and young people experiencing intellectual disabilities, these narrowly focus on disease or health conditions. The objective of this planned systematic review is therefore to collate, summarise and critically appraise the psychometric properties of health and wellbeing measures used with adolescents (aged 11-16) with an intellectual disability.

\section{Methods}

We designed and registered a study protocol for a systematic review of studies which explores the psychometric properties of health and wellbeing measures used with adolescents experiencing intellectual disabilities. Electronic databases including PsycINFO, CINAHL, MEDLINE and ERIC will be searched using predefined search terms to identify relevant studies. Quantitative and mixed-methods studies, and studies published in peer-reviewed journals or grey literature will be included. Review papers, editorials and case studies will be excluded. Eligible studies should identify self and/or proxy reported health and wellbeing measures which assess health and wellbeing among adolescents with intellectual disabilities. The methodological quality of the included studies will be assessed by applying the COSMIN Risk of Bias Checklist. The quality of the evidence (i.e., the total body of evidence used for the overall ratings on each psychometric property of an instrument) will be evaluated in accordance with the GRADE guidelines.

\section{Discussion}

This systematic review will be among the first to systematically explore the psychometric properties of health and wellbeing measures used with adolescents experiencing intellectual disabilities. By providing evidence-based knowledge about measures being used in health and wellbeing research amongst this population, and more importantly how reliable and valid these measures are, the most suitable for use will be identified. Our findings will be of potential interest to clinicians, researchers and service providers who need information about the methodological quality and the characteristics of measures to make informed decisions about the most reliable and valid tool for a specific purpose. The findings from this study will contribute to the knowledge surrounding available and appropriate measures to use for measuring the health and wellbeing of adolescents with intellectual disabilities, which are necessary to inform intervention development and future health policy.

\section{Systematic Review Registration}


The protocol has been registered at the International Prospective Register of Systematic Reviews (PROSPERO). The registration number is: CRD42021231697.

\section{Background}

Wellbeing can be understood as how people feel and function, both on a personal and social level, and how they evaluate their lives as a whole (New Economic Foundation, 2012). The concept of wellbeing has been defined and operationalised in many ways and tends to encompass both objective (e.g., income, education, and health) and subjective (e.g., happiness, perception of quality of life and life satisfaction) aspects of a person's life (Statham \& Chase 2010). According to the World Health Organisation (WHO, 2021), health is a state of complete physical, mental, and social wellbeing, not merely the absence of disease or infirmity. The WHO definition links health explicitly with wellbeing and conceptualises health as a human right requiring physical and social resources to achieve and maintain. In recent years, the concept of children's wellbeing has become firmly embedded in discourse and policy in the academic, government and public sectors in countries across the world (e.g., Statham \& Chase 2010). Since the publication of UNICEF's Report Card 7 (2007) entitled 'Child poverty in perspective: An overview of child wellbeing in rich countries' children's wellbeing has been put firmly on the agenda in many countries around the world (UNICEF, 2007).

Wellbeing is a particularly important concept for children and young people with intellectual disabilities (ID). An ID is a disability characterised by significant limitations in both intellectual functioning and in adaptive behaviour, as expressed in conceptual, social, and practical adaptive skills (AAIDD, 2010). It is a generalised disorder, which encompasses a wide array of cognitive and behavioural deficiencies; and affects the individuals in all areas of life (AAIDD, 2010). The severity of ID is classified into four types, based on an intelligence quotient (IQ) test, namely mild, moderate, severe, and profound ID (AAIDD 2010). Children and adolescents with an ID are more likely to experience diminished wellness as they age, as well as having an increasing number of chronic conditions and lower socioeconomic status than their non-disabled peers (Menear et al., 2015).

Whilst there is a wide range of research examining adolescent's health and wellbeing, few of these studies include children and young people with ID (Boström \& Broberg, 2018; Davidson et al., 2017). Current large-scale global studies of wellbeing such as the WHO Health Behaviour of School-aged Children (HBSC) and Children's Worlds do not include children who experience ID. This is of particular concern given that individuals with ID tend to experience poorer health, as well as score lower on a number of health and wellbeing indicators in comparison to their typically developed peers (Emerson, 2011). Many health and wellbeing measures are usually administered by survey and are not accessible to youth with ID. This population may have difficulty understanding the format and complexity of questions and response scales used in questionnaires designed for same-age peers (Boström et al., 2016; Davidson et al., 2017), as intellectual impairment is often associated with difficulties in communication (Shevell, 2008), working memory (Lifshitz et al., 2011) and self-insight (Jahoda et al., 2010), all of which are required to respond effectively to questionnaires. In 2011, Scott and colleagues carried out a pilot study 
including children (aged 11-17 years) with ID/Special Educational Needs in the WHO HBSC Questionnaire. Using the standard protocol, just over half of the children were able to 'complete' the survey questionnaire, however a high proportion of their responses proved to be uncodable (Scott et al., 2011).

Children and young people with ID have traditionally been assessed via proxy reports completed by parents, teachers, or carers as it is assumed that these young persons do not have the capacity to selfreport on these domains (Shevell, 2008). Thus, a notable limitation is that a third party is reporting on the wellbeing of the person with an ID (Hayes et al., 2013). More broadly, since measuring health and wellbeing involves a subjective perspective, the use of proxy-reports to determine health and wellbeing has been questioned, and it has been concluded that self-reports cannot simply be replaced by proxyreports (Verdugo et al., 2005). It is now appreciated that the young person's view should, where possible, be sought directly rather than being inferred from proxy-reports. However, proxy-reports should not necessarily be discarded, but rather employed as an additional source of information to an individual's health and wellbeing (Clark et al., 2015; Egilson et al., 2017; Knüppel et al., 2018), as proxy-reports can be useful when used in addition to child self-reports to assess multiple perspectives (Davidson et al., 2017).

Authors repeatedly note the under-representation of young people with ID in health and wellbeing surveys (Hicks et al., 2011; Selwn \& Riley, 2015). A report from the Children's Worlds survey highlights the exclusion of marginalised children, including those in special educational needs settings, and suggests that there is a real need for ways to be found to "include these children in research on children's lives and wellbeing" (Rees and Main 2015:16). Research has demonstrated that many children with ID do have the capacity to respond to self-report questionnaires (Douma et al., 2006; Emerson et al., 2005) and that adaptations such as pictorial representations and limited forced choice options can strengthen their validity (Hartley and MacLean, 2006). Thus, there has been an emphasis on finding alternative and more accessible ways to enable this population to complete questionnaires independently, reliably, and confidentially (Boström \& Eriksson, 2015; Boström at el., 2016). Increasing attention has been paid to the involvement of children and young people with ID in the design of wellbeing measures and capturing their views on what wellbeing means to them and what is important to their quality of life (Hicks et al., 2011). For example, Boström and colleagues (2016) developed the Wellbeing in Special Education Questionnaire (WellSEQ) in cooperation with 113 students (aged 12-16) and teachers in special educational needs settings for children with cognitive disabilities (Boström et al., 2016). Both students and teachers participated in the process of cooperative inquiry (Frauenberger et al., 2011) designing and developing the research instruments in three workshops using different methods (brainstorming, sketching, paperprototyping, focus group, direct observations, and existing system analysis) (Boström \& Eriksson, 2015; Boström et al., 2016). The WellSEQ is specifically tailored to measure self-rated mental and ill health, peer relations and school and family environment in adolescents with mild or moderate intellectual and developmental disability and is administered via an interactive touch-based application for tablet PCs (Boström et al., 2016). The standard method for collecting reliable self-reports from persons with ID has consisted of structured interviews (e.g., Emerson, 2005; Haynes et al., 2013), but because this is a highly time-consuming procedure, there has been a shift of focus to design questionnaires specifically for this 
population. As a result, in the past decade, and in line with the trend of stimulating participatory research in people with IDs, there seems to be an increase in studies using self-reports to assess the health and wellbeing of adolescents with ID.

Researchers have starting to address the dearth of instruments suitable for measuring health and wellbeing with adolescents in the ID population. Whilst there are studies (e.g., Carlon et al., 2010; Jardine et al., 2014; Morris et al., 2014; Solans et al., 2008) that have systematically reviewed the psychometric properties of QoL measures for children and young people with ID, these narrowly focus on disease or health conditions (Davidson et al., 2017). Therefore, an up-to-date systematic review is warranted to summarize and appraise the psychometric properties of health and wellbeing measures focused on adolescents with ID as they are useful in selecting a measure that is fit for purpose (Mason at al., 2018; Scholtes et al., 2011). This review will provide evidence-based knowledge regarding what measures are being used in health and wellbeing research amongst this population, and more importantly how reliable and valid these measures are. The most suitable health and wellbeing measures for use amongst adolescents with ID will be identified, and recommendations will be gathered to determine their reliability (i.e., to identify what works). This is urgently needed as clinicians, researchers and service providers require information about the methodological quality and the characteristics of measures to make informed decisions about the most reliable and valid tool for a specific purpose (Kipfer and Pihet, 2019). It is expected that this systematic review will assist in informing choice when selecting an instrument for the measurement of health and wellbeing with this population. It will contribute to the knowledge surrounding available and appropriate measures to use for measuring the health and wellbeing of adolescents with ID, which are necessary to inform intervention development and future health policy.

The aim of this systematic review is to collate, summarise and critically appraise the psychometric properties of health and wellbeing measures used with children and young people (aged 11-16) with ID. The proposed systematic review will answer the following questions:

1. What measures are being/have been used to assess health and wellbeing of adolescents with ID?

2. What is the methodological quality of these health and wellbeing measures for use with adolescents with ID?

3. What measures work best for assessing health and wellbeing of adolescents with ID?

\section{Methods}

Studies included in the systematic review will use the Patient/Problem, Intervention Comparison, Outcome and Time or PICOT (Fineout-Overholt and Johnson, 2005) mnemonics for reviews. PICOT based eligibility and inclusion criteria are described in Table 1. 
Table 1

Patient/Problem, Intervention Comparison, Outcome and Time (PICOT) Research Strategy for Systematic Review

\section{PICOT Description}

(P) Patient $\quad$ Adolescents (both male and female) aged 11-16 years with a diagnosed ID (IQ > population 70)

(I) Intervention Reviewing the psychometric properties of both self and proxy reported measures or Interest which assess health and wellbeing among adolescents with ID

(C) Not applicable

Comparison

interventions

(0) Outcomes:

Primary outcomes

Secondary outcomes

( $\mathrm{T}$ ) Time

Other considerations
Identify instruments used in ID population

Determine psychometric properties of health and wellbeing measures

Studies published from January 2000 to February 2021 will be considered to ensure we focus on old and new instruments used in the past 20 years.

Age limits: adolescents in the age group 11-16

Language: English

Study design: quantitative and mixed methods

The present protocol has been written according to the PRISMA-P (Preferred Reporting Items for Systematic Reviews and Meta-Analyses) guidelines (Moher et al., 2016) (see PRISMA-P checklist is provided in Supplemental File 1). This protocol has been registered at the International Prospective Register of Systematic Reviews (PROSPERO) database (registration number: CRD42021231697).

\section{Search Strategy}

The search strategy was developed after consultation with Ulster University's Faculty of Life and Health Sciences librarian. The search strategy is broad to include all research articles that use a psychometric instrument to measure health and wellbeing among adolescents with ID.

A systematic database search was performed using the Psychological Information Database (PsycINFO), Cumulative Index of Nursing and Allied Health Literature (CINAHL), Medical Literature Analysis Retrieval System Online (MEDLINE), and Education Resources Information Center (ERIC). Key terms pertaining to intellectual disability, health and wellbeing, psychometric properties, measures, and adolescence were used to search for studies for the review (see Supplemental File 2 for the search strategy and full search 
strings for each of the five-key concepts in each database). Studies published from January 2000 to March 2021 and available in the English language will be considered.

Quantitative and mixed-methods studies, and studies published in peer-reviewed journals or grey literature will be included. Grey literature will be searched for additional reports and papers. Using our search terms web searches Google and Google Scholar, and websites of any charitable and nongovernmental organisations will be searched to obtain relevant reports i.e., Barnardo's, Save the Children etc. EThOS (e-thesis online service) will also be used to search for thesis's and dissertations relevant to our study. Review papers, editorials, or case studies will be excluded.

Eligible studies should report instruments designed for use with children or adolescents (aged 11-16) with ID to assess their general, subjective and/or objective health and wellbeing, including concepts such as health-related quality of life, life satisfaction, happiness, and wellness. Studies designed for adults or older people with ID, and studies that do not assess health and wellbeing domains will be excluded. Studies from all countries will be included as long as they are available in an English translation. Studies that have not been translated into English will be excluded.

The study population will include adolescents between the ages of 11 and 16 years. If a study includes a broader age range encompassing 11-16-year-olds, and if it is possible, data will be extracted for only the targeted age groups. Participants must have an ID diagnosis (mild, moderate, severe, or profound). Young children (aged 10 or younger), young adults (17 years or above) and elderly people (over 70 ). Studies will be excluded if it is not clear whether participants have an intellectual disability, or where they have other conditions (i.e., autism, epilepsy, or physical disabilities) without specifically noting that they also have an intellectual disability.

\section{Data Extraction}

Data will be extracted as follows.

Literature Search and Study Selection

Titles and/or abstracts of studies retrieved from the database searches and those from additional sources will be screened independently in Covidence by two review authors (SM and JD) to identify studies that meet the inclusion criteria outlined above. The full text of these potentially eligible studies will be retrieved and independently assessed for eligibility by two review authors. Any disagreement between them over the eligibility of studies will be resolved through discussion with a third review author (MM). The interrater agreement will be assessed by calculating weighted k (Cohen \& Humphreys, 1968) and interpreted as very good (0.81-1.00), good (0.61-0.80), moderate $(0.41-0.60)$, fair $(0.21-0.40)$, and poor (0.00-0.20( (Altman, 1991).

A standardised, pre-piloted form will be used to extract data from the selected studies for assessment of study quality and evidence synthesis. Two review authors will record information related to: (1) study characteristics (i.e., study setting; study population, study sample (n); study methodology) (2) instrument 
characteristics (i.e., reporter (self and/or proxy); type of instrument; format of the instrument (i.e., number of items and response formats), psychometric properties of the instrument (e.g., Cronbach's alpha, reliability analysis); and the results of the measure/study). Where needed, we will contact authors for original data with a maximum of three contact emails: once a month over three months.

\section{Study Quality Assessment \& Data Synthesis Evaluation of Methodological Quality of Studies}

Studies evaluating the measurement properties of an assessment require high methodological quality with a low-risk bias to guarantee that appropriate conclusions are drawn about the properties of the measure (Terwee et al., 2009). Thus, it is important to evaluate those methodological qualities (De Vet et al., 2011). Two review authors will assess the methodological quality by applying the COnsensus-Based Standards for the Selection of Health Status Measurement INstruments (COSMIN) Risk of Bias Checklist (Mokkink et al., 2018). The COSMIN Checklist contains ten boxes with standards for PROM development (box 1) and for nine measurement properties: including content validity (box 2); structural validity (box 3); internal consistency (box 4); cross-cultural validity $\backslash$ measurement invariance (box 5); reliability (box 6); measurement error (box 7); criterion validity (box 8); hypotheses testing for construct validity (box 9); and responsiveness (box 10) (Mokkink et al., 2018). Only checklist items that are assessed in each included study will be completed since not all measurement properties are evaluated in all articles. For each study, an overall judgement will be provided on the quality of the particular study. We will use a four-point rating system where each standard within a COSMIN box will be rated as 4 = 'very good', 3 = 'adequate', 2 = 'doubtful' or 1 = 'inadequate' (Mokkink et al., 2018). The total score of methodological quality ratings per psychometric property will be presented as a percentage of the ratings: inadequate $(0-25 \%)$; doubtful (25.1-50\%); adequate (50.1-75\%); and very good (75.1-100\%). Two review authors will rate the methodological quality independently, and any discrepancies will be resolved by consensus.

\section{Evaluation of Psychometric Properties of Instruments}

The result of each single study on a measurement property will be rated against the updated criteria for good measurement properties (Terwee et al., 2007) on which consensus will be achieved by the two review authors. Each result will be rated as either: sufficient (above the quality criteria threshold: + ); insufficient (below the quality criteria threshold: -); or indeterminate (less robust data that do not meet the quality criteria: ?) using the predefined criteria for good psychometric properties (Mokkink, et al., 2018). A $75 \%$ agreement rate will be used (Mokkink et al., 2018), that is for an overall sufficient (+) or insufficient $(-)$ rating on a psychometric property, $75 \%$ or more of the studies reporting the psychometric property must be sufficient $(+)$ or insufficient (-). Otherwise, for an overall inconsistent $( \pm$ ) rating, less than $75 \%$ of studies will show the same rating, and for overall indeterminate (?) rating, all studies will be indeterminate (?).

The evidence will be summarised, and the quality of the evidence (i.e., the total body of evidence used for the overall ratings on each psychometric property of an instrument) will be graded as high, moderate, low, 
or very low using the Grading of Recommendations, Assessment, Development and Evaluations (GRADE) guidelines (see Prinsen et al., 2018).

\section{Selection of Instruments}

The selection of instruments and recommendation of suitable instruments for future use will be based on a combining overall rating results of each psychometric property (Step 2) and grading results (Step 3) (Prinsen et al., 2018). Each instrument will be classified into three recommendation categories (Mokkink et al., 2018): (A) most suitable (i.e., instruments with high-quality evidence for sufficient content validity in any aspects of relevance, comprehensiveness, and comprehensibility, and at least low-quality evidence for sufficient internal consistency); (B) promising but need further validation studies (i.e., instruments categorized not in $A$ or $C$ ); and (C) not recommendable (i.e., instruments with high quality evidence for an insufficient psychometric property).

\section{Discussion}

To the best of our knowledge, this is the first systematic review exploring the psychometric properties of health and wellbeing measures used with adolescents experiencing intellectual disabilities. By providing evidence-based knowledge about measures being used in health and wellbeing research amongst this population, and more importantly how reliable and valid these measures are, the most suitable for use will be identified. This is crucial as clinicians, researchers and service providers need information about the methodological quality and the characteristics of measures to make informed decisions about the most reliable and valid tool for a specific purpose. The findings of this study will contribute to the knowledge surrounding available and appropriate measures to use for measuring the health and wellbeing of adolescents with intellectual disabilities, which are necessary to inform intervention development and future health policy.

One limitation of the systematic review process is the exclusion of studies that are not available in the English language, which may mean that articles identifying health and wellbeing measures used with adolescents experiencing intellectual disabilities based in non-English speaking countries and not available in the English language will be excluded.

\section{Abbreviations}

ID

Intellectual Disability; COSMIN:COnsensus-Based Standards for the Selection of Health Status Measurement Instruments; PsycINFO:Psychological Information; CINAHL:Cumulative Index of Nursing and Allied Health Literature; MEDLINE:Medical Literature Analysis Retrieval System Online; ERIC:Education Resources Information Center; GRADE:Grading of Recommendations, Assessment, Development and Evaluations; PRISMA-P:Preferred Reporting Items for Systematic Reviews and MetaAnalyses. 


\section{Declarations}

\section{Ethics approval and consent to participate.}

Ethical approval is not required for this systematic review as no tests, measurements or experiments were performed on humans as part of this work.

\section{Consent for publication}

Not applicable.

\section{Availability of data and materials}

The studies included in the review will be available from the corresponding author upon request.

\section{Competing interests}

The authors declare no competing interests.

\section{Funding}

A Baily Thomas Charitable Fund (TRUST/VC/AC/SG/5662-8764) funded SM's research assistance. The funders did not fund protocol development and are not responsible for the content of the review protocol.

\section{Author's Contributions}

SM led the conceptualisation of the protocol with input from all authors. All authors have read and approved the final manuscript.

\section{Acknowledgements}

Not applicable.

\section{References}

1. American Association on Intellectual Developmental Disabilities (AAIDD). Intellectual disability: Definition, classification, and systems of supports. Washington, DC: AAIDD; 2010.

2. Altman, DG. Practical statistics for medical research. Chapman and Hall; 1991.

3. Boström, P \& Broberg, M. Protection and restriction: A mixed- methods study of self- reported wellbeing among youth with intellectual disabilities. Journal of Applied Research in Intellectual Disabilities. 2018; 31:1.

4. Boström, P \& Eriksson, E. Design for self-reporting psychological health in children with intellectual disabilities. Presented at the Interaction design and children, Boston, MA, USA; 2015. 
5. Boström P, Johnels JÅ, Thorson M \& Broberg M. Subjective mental health, peer relations, family, and school environment in adolescents with intellectual developmental disorder: a first report of a new questionnaire administered on tablet PCs. Journal of Mental Health Research in Intellectual Disabilities. 2016;9: 207-31.

6. Carlon S, Shields N, Yong K, Gilmore R., Sakzewski L \& Boyd R. A systematic review of the psychometric properties of quality-of-life measures for school aged children with cerebral palsy, BMC Paediatrics. 2010; 81:1-11.

7. Clark BG, Magill-Evans JE \& Koning C. Youth with autism spectrum disorders: self- and proxyreported quality of life and adaptive functioning. Focus on Autism and Other Developmental Disabilities. 2015; 30:57-64.

8. Cohen J, \& Humphreys LH. Weighted Kappa: Nominal scale agreement provision for scaled disagreement or partial credit. Psychological Bulletin. 1968;4: 213-20.

9. Davidson G, Irvine R, Corman M, Kee F, Kelly B, Leavey G, \& McNamee C. Measuring the Quality of Life of People with Disabilities and their Families: Scoping Study Final Report. Department for Communities. 2017. https://www.communities-ni.gov.uk/publications/measuring-quality-lifedisabled-people-and-theirfamilies-scoping-study-final-report. Accessed 22 Jan 2021.

10. De Vet HC, Terwee CB, Mokkink LB, \& Knol DL. Systematic reviews of measurement properties. In: De Vet HCW, Terwee CB, Mokkink LB, Knol DL, editors. Measurement in Medicine: A practical guide. New York: Cambridge University Press; 2011. p.275-314.

11. Douma, JCH, Dekker MC, Verhulst FC, \& Koot HM. Self-reports on mental health problems of youth with moderate to borderline intellectual disabilities. Journal of the American Academy of Child and Adolescent Psychiatry, 2006;10: 1224-1231.

12. Egilson ST, Ólafsdóttir, LB, Leósdóttir, T, \& Saemundsen E. (2017). Quality of life of high-functioning children and youth with autism spectrum disorder and typically developing peers: Self- and proxyreports. Autism. 2017;2: 133-141.

13. Emerson E. Health Status and Health Risks of the "Hidden Majority" of Adults With Intellectual Disability. Intellectual \& Developmental Disabilities, 2011; 49: 155-165. doi: https://doi.org/10.1352/1934-9556-.

14. Emerson E, Robertson J, \& Wood J. Emotional and behavioral needs of children and adolescents with intellectual disabilities in an urban conurbation. Journal of Intellectual Disability Research. 2005;49: 16-24.

15. Frauenberger C, Good J, \& Keay-Bright W. Designing technology for children with special needs: Bridging perspectives through participatory design. CoDesign, 2011; doi:10.1080/15710882.2011.587013.

16. Fineout-Overholt E., \& Johnson, L. Teaching EBP: Asking searchable, answerable clinical questions. Worldviews on Evidence-Based Nursing. 2005;3: 157-60; doi: 10.1111/j.1741- 6787.2005.00032.

17. Hartley S L, \& MacLean WE. A review of the reliability and validity of Likert-type scales for people with intellectual disability. Journal of Intellectual Disability Research. 2006;50: 813-827. 
18. Hicks S, Newton J, Haynes J \& Evans, J. Measuring children's and young people's well-being. Cardiff: Office for National Statistics and BRASS, Cardiff University;2011.

19. Jahoda A, Wilson A, Stalker K, \& Cairney A. Living with stigma and the self-perceptions of people with mild intellectual disabilities. Journal of Social Issues. 2010; 66: 521-534.

20. Jardine J, Glinianaia S, McConachie H, Embleton, ND, \& Rankin J. Self-reported quality of life of young children with conditions from early infancy: A systematic review, Paediatrics. 2014;4: 11291148.

21. Kipfer S, \& Pihet S. Reliability, validity and relevance of needs assessment instruments for informal dementia caregivers: a psychometric systematic review. JBI Database System Rev Implement Rep. 2019; doi: 10.11124/JBISRIR-2017-003976.

22. Knüppel A, Telléus GK, Jakobsen H, Lauritsen MB. Quality of life in adolescents and adults with autism spectrum disorder: Results from a nationwide Danish survey using self-reports and parental proxy-reports. Res Dev Disabil. 2018; doi: 10.1016/j.ridd.2018.09.004.

23. Lifshitz H, Shtein S, Weiss I, \& Vakil, E. Meta-analysis of explicit memory studies in populations with intellectual disability. European Journal of Special Needs Education. 2011;26: 93-111.

24. Mason SJ, Catto JWF, Downing A, Bottomley SE, Glaser AW, Wright P. Evaluating patient-reported outcome measures (PROMs) for bladder cancer: a systematic review using the COnsensus-based Standards for the selection of health Measurement INstruments (COSMIN) checklist. BJU Int. 2018; doi: $10.1111 /$ bju. 14368 .

25. Menear KS, Preskitt JK, Goldfarb SS, \& Menachemi N. Correlates of wellness among youth with functional disabilities. Disabil Health J. 2015;2: 223-30.

26. Moher D, Stewart L \& Shekelle P. Implementing PRISMA-P: recommendations for prospective authors. Systematic Reviews, 2016;5:15.

27. Mokkink LB, Vet HC, Prinsen CA, Patrick D, Alonso J, Bouter LM, et al. COSMIN Risk of Bias checklist for systematic reviews of Patient-Reported Outcome Measures. Qual Life Res. 2018.

28. Mokkink LB, Prinsen, CAC, Patrick DL, Alonso J, Bouter LM, de Vet HCW, \& Terwee, CB. COSMIN methodology for systematic reviews of Patient-Reported Outcome Measures (PROMs)-User manual (version 1.0) 2018.

29. Morris C, Janssens A, Allard A, Thompson Coon J, Shilling V, Tomlinson R, Williams J, Fellowes A, Rogers M, Allen K, Beresford B, Green C, Jenkinson C, Tennant A. \& Logan S. Informing the NHS Outcomes Framework: evaluating meaningful health outcomes for children with neurodisability using multiple methods including systematic review, qualitative research, Delphi survey and consensus meeting. Health Services and Deliver Research, 2014;15.

30. New Economic Foundation. Measuring Wellbeing: A guide for practitioners. London: New Economics Foundation; 2012.

31. Prinsen C AC, Mokkink LB, Bouter LM, Alonso J, Patrick DL, de Vet HCW, \& Terwee CB. COSMIN guideline for systematic reviews of patient-reported outcome measures. Quality of Life Research. 2018:5; 1147-1157. 
32. Rees G \& Main G. Children's views on their lives and well-being in 15 countries: An initial report on the Children's Worlds survey, 2013-14. York, UK: Children's Worlds Project; 2015.

33. Selwyn J \& Riley S. Measuring well-being: A literature review. Bristol: Hadley Centre for Adoption and Foster Care Studies \& Coram Voice; 2015

34. Scholtes VA, Terwee CB, Poolman RW. What makes a measurement instrument valid and reliable? Injury. 2010. doi: 10.1016/j.injury.2010.11.042.

35. Scott J, Wishart J \& Currie C. Including Children with Intellectual Disabilities/Special Educational Needs into National Child Health Surveys: A Pilot Study. Journal of Applied Research in Intellectual Disabilities. 2011:24. doi: 10.1111/j.1468-3148.2010.00621.x.

36. Shevell, M. Global developmental delay and mental retardation or intellectual disability: Conceptualisation evaluation and etiology. Paediatric Clinics of North America. 2008;55: 1071-1084.

37. Solans M, Pane S, Estrada M, Serra-Sutton V, Berra S, Herdman M, ALonso J \& Rajmil L. Healthrelated quality of life measurement in children and adolescents: A systematic review of generic and disease-specific instruments, Value in Health. 2008;4: 742-764.

38. Statham J \& Chase E. Child wellbeing: a brief overview. Childhood Wellbeing Research Centre Briefing Paper. London: Institute of Education. 2010 http://www.navo.org.uk/downloads/documents/FinalChildDFEwebsite.pdf

39. Terwee CB, Mokkink LB, Knol DL, Ostelo RWJG, Bouter LM, De Vet HCW. Rating the methodological quality in systematic reviews of studies on measurement properties: a scoring system for the COSMIN checklist. Qual Life Res. 2012; 21:651-7.

40. Terwee CB, Jansma EP, Riphagen II, de Vet HC. Development of a methodological PubMed search filter for finding studies on measurement properties of measurement instruments. Qual Life Res. 2009;18.

41. Terwee C, Bot S, de Boer M, van der Windt D, Knol D, Dekker J, et al. Quality criteria were proposed for measurement properties of health status questionnaires. J Clin Epidemiol. 2007;60: 34-42.

42. UNICEF. Child poverty in perspective: An overview of child wellbeing in rich countries. A comprehensive assessment of the lives and wellbeing of children and asolescents in the economically advanced nations. Report Card 7. UNICEF Innocenti Research Centre, Florence. 2007. https://www.unicef.org/media/files/ChildPovertyReport.pdf. Accessed 21 Jan 2021.

43. Verdugo M.A, Schalock, RL, Keith KD \& Stancliffe RJ Quality of life and its measurement: Important principles and guidelines. Journal of intellectual disability research. 2005;10: 707-717.

44. WHO Constitution of the World Health Organisation. https://www.who.int/about/who-weare/constitution (2021) Accessed 21 Feb 2021.

\section{Supplementary Files}

This is a list of supplementary files associated with this preprint. Click to download. 
- AdditionalFile1PRISMAPchecklist.docx

- AdditionalFile2SearchStrategy.docx 